\title{
gु
}

\section{Fourier-Transform Ghost Imaging with Hard X Rays}

\author{
Hong Yu, ${ }^{1}$ Ronghua Lu, ${ }^{1}$ Shensheng Han, ${ }^{1, *}$ Honglan Xie, ${ }^{2}$ Guohao Du, ${ }^{2}$ Tiqiao Xiao, ${ }^{2}$ and Daming Zhu ${ }^{3,4}$ \\ ${ }^{1}$ Key Laboratory for Quantum Optics and Centre for Cold Atom Physics, Shanghai Institute of Optics and Fine Mechanics, \\ Chinese Academy of Sciences, Shanghai 201800, China \\ ${ }^{2}$ Shanghai Institute of Applied Physics, Chinese Academy of Sciences, Shanghai 201800, China \\ ${ }^{3}$ University of Science and Technology of China, Hefei 230026, China \\ ${ }^{4}$ University of Missouri-Kansas City, Kansas City, Missouri 64110, USA
}

(Received 13 May 2016; published 7 September 2016)

\begin{abstract}
Knowledge gained through x-ray crystallography fostered structural determination of materials and greatly facilitated the development of modern science and technology in the past century. However, it is only applied to crystalline structures and cannot resolve noncrystalline materials. Here we demonstrate a novel lensless Fourier-transform ghost imaging method with pseudothermal hard x rays that extends x-ray crystallography to noncrystalline samples. By measuring the second-order intensity correlation function of the light, Fourier-transform diffraction pattern of a complex amplitude sample is achieved at the Fresnel region in our experiment and the amplitude and phase distributions of the sample in the spatial domain are retrieved successfully. For the first time, ghost imaging is experimentally realized with $x$ rays. Since a highly coherent x-ray source is not required, the method can be implemented with laboratory x-ray sources and it also provides a potential solution for lensless diffraction imaging with fermions, such as neutrons and electrons where intensive coherent sources usually are not available.
\end{abstract}

DOI: 10.1103/PhysRevLett.117.113901

Since Max Laue discovered $x$-ray diffraction in crystals in 1914, x-ray crystallography has become a powerful tool in exploring and analyzing the internal structures of complex materials, such as biomolecular structures and nanomaterials [1-3]. However, the structure information of many important molecular materials, such as membrane proteins, is still out of reach, because these materials are difficult to grow into macroscopic crystals. Furthermore, with the rapid development of nanoscience and biology, it has become an urgent need to obtain images of the internal structure of samples in their natural states instead of in crystals. In 1999, the coherent diffraction imaging (CDI) method [4-6] was proposed to extend $\mathrm{x}$-ray crystallography to allow imaging noncrystalline structures in nanoscale by illuminating the samples with coherent $\mathrm{x}$ rays and recording the diffraction patterns in the far field. Fresnel CDI $[7,8]$ and ptychography techniques $[9,10]$ were proposed to circumvent the intrinsic restriction of sample size in classical CDI. Huge progress has been made with nanocrystals at $\mathrm{x}$-ray free electron lasers $[11,12]$. Nevertheless, in classical CDI, the recorded diffraction pattern within the beamstop is missing, and additional technology is needed for low-frequency information. Most of all, due to the requirements for high coherence and brightness, synchrotron radiation or x-ray free electron laser sources are still essential to x-ray CDI applications, and high quality imaging using laboratory $\mathrm{x}$-ray sources with CDI techniques remains to be achieved.

Most of the conventional x-ray imaging methods are based on detection of intensity distribution of light fields, i.e., the first-order correlation of the light. In fact, imaging in both real and reciprocal space can be realized with thermal light through the ghost imaging technique, a phenomenon first observed in the quantum regime $[13,14]$ by measuring higher order correlation of light fields. Different from conventional methods, in a typical ghost imaging system, the light field passing through or reflected by a sample is recorded only with a nonspatially resolving detector (i.e., a bucket or point detector), and the sample's information is acquired from the second-order intensity fluctuation correlation of the scattered and unscattered lights. Ghost imaging has been proved and demonstrated with classical visible thermal light [15-21] and applied quickly in remote sensing, photolithography, superresolution imaging, single-pixel three dimensional cameras, etc. [22-33]. A lensless ghost imaging scheme $[17,34]$ with spatially incoherent illumination, where the Fourier-transform diffraction pattern of the sample can be acquired at the Fresnel region, had also been proposed to achieve the same diffraction pattern as in CDI. Therefore, this Fourier-transform ghost imaging method provides a possibility to achieve images of noncrystalline samples with incoherent X-ray sources. However, a beam splitter is needed in the scheme to generate two copies of the incident light field, which is easy for visible light but difficult for $\mathrm{x}$ rays.

In this Letter, we report for the first time the success of an experiment which demonstrates hard x-ray Fouriertransform ghost imaging (FGI) using a novel experimental approach. The experiment was based on the same principle of visible light FGI which is illustrated in Fig. 1(a). A light beam from a spatially incoherent source is divided into two beams, a testing beam and a reference beam, after passing through a 
beam splitter. The light in the testing beam passes through a sample at a distance $d_{1}$ and then is recorded by a point detector $D_{t}$ positioned at a distance $d_{2}$ from the sample. The reference beam does not pass through the sample at all, but its intensity distribution after passing through a distance $d$ is recorded by a spatially resolved panel detector $D_{r}$.

As the derivations detailed in Ref. [17] show, under the condition where $d_{1}+d_{2}=d$, the correlation function between the intensity fluctuation of reference beam $\Delta I_{r}\left(x_{r}\right)$ and that of the testing beam $\Delta I_{t}\left(x_{t}\right)$ measured by the two detectors is directly related to the modulus of the Fourier transformation of the sample's transmittance, and such a relation can be expressed as

$$
\left\langle\Delta I_{r}\left(x_{r}\right) \Delta I_{t}\left(x_{t}\right)\right\rangle=\frac{I_{0}^{2}}{\lambda^{4} d_{2}^{4}}\left|T\left(\frac{2 \pi\left(x_{t}-x_{r}\right)}{\lambda d_{2}}\right)\right|^{2},
$$

where $\Delta I_{k}\left(x_{k}\right)=I_{k}\left(x_{k}\right)-\left\langle I_{k}\left(x_{k}\right)\right\rangle, k=r$ or $t, x_{r}$ and $x_{t}$ are the coordinates at the detector planes in the reference and testing beams, respectively, $\lambda$ is the wavelength, $I_{0}$ is the intensity of the incident light, and $T\left[2 \pi\left(x_{t}-x_{r}\right) / \lambda d_{2}\right]$ is the Fourier transformation of the sample's transmittance. Real space image of the sample can be retrieved from $\left|T\left[2 \pi\left(x_{t}-x_{r}\right) / \lambda d_{2}\right]\right|$ as in conventional x-ray CDI [34]. Thus, the sample can be imaged by recording the incoherent spatial intensity distribution of the beam without passing through the sample and then correlated with the signal detected by a single pixel detector placed behind the sample. Such an imaging method, which has been intensively studied using a visible pseudothermal light source [19,34], removes the need for a beam stop and avoids missing low-frequency data in the diffraction patterns as in traditional x-ray CDI.

However, to realize FGI in the hard $\mathrm{x}$-ray regime is challenging. For hard $\mathrm{x}$ rays, there is no perfect beam splitter which can produce twin beams as is the case with visible light [35]. To circumvent such a difficulty, we notice that the condition $d_{1}+d_{2}=d$ is satisfied in the scheme. So, for a controllable pseudothermal light source, rather than splitting the thermal light into two beams as in Fig. 1(a), we can use an equivalent scheme as shown in Fig. 1(b), which uses only one spatially incoherent pseudothermal light beam and a fixed panel detector by shuttling the sample in and out of the beam in one pseudocoherent duration (during which the light source is stable). When the sample is inserted into the beam, the signals detected by a single pixel of the panel detector serve as that detected with the point detector in the testing beam, when the sample is moved out of the beam, the signals detected serve as that detected by the panel detector in the reference beam, so both the intensity fluctuation $\Delta I_{t}\left(x_{t}\right)$ and $\Delta I_{r}\left(x_{r}\right)$ can be acquired by the same panel detector if only the two measurements are completed in one pseudocoherent duration.

The experiment was performed on the $13 \mathrm{~W}$ beam line at the Shanghai Synchrotron Radiation Facility (SSRF), which is dedicated to $\mathrm{x}$-ray imaging and biomedical applications.
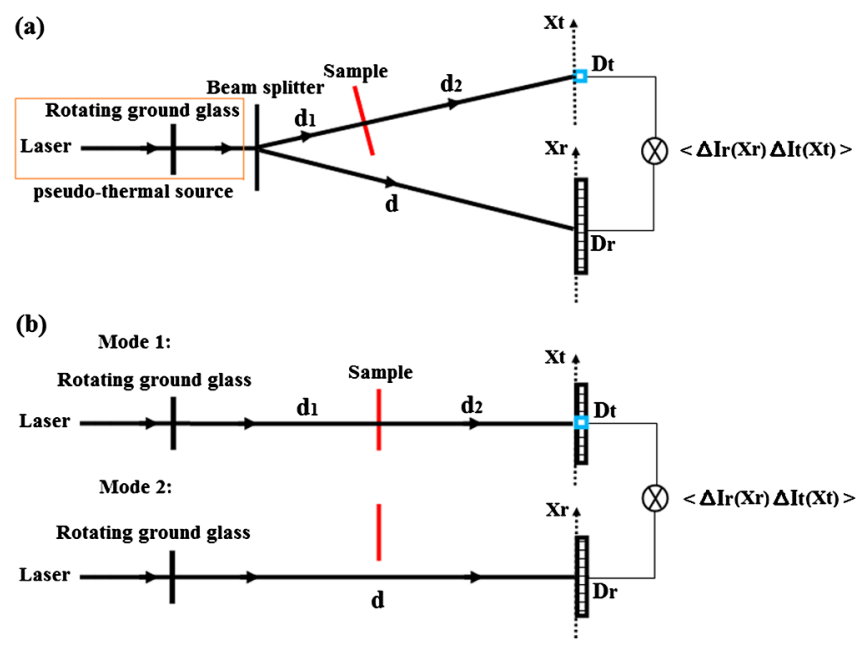

FIG. 1. Illustration of the experimental setup for FGI without a beam splitter. (a) The principle of lensless FGI and (b) the setup of a lensless FGI system without a beam splitter: For a controllable pseudothermal light source, when the sample is inserted into the light beam as in mode 1, each pixel of the panel detector can be considered as a point detector $D_{t}$ in the testing beam of FGI, while when the sample is moved out of the beam as in mode 2, the panel detector records the intensity distribution without passing through the sample as the reference beam detector $D_{r}$ of FGI, if only the two mode measurements are completed in one pseudocoherent duration determined by the rotating speed of the ground glass.

Figure 2(a) shows the experimental setup. A pseudothermal $\mathrm{x}$-ray source [36-38], which can generate a controllable chaotic $\mathrm{x}$-ray speckle pattern fluctuation to emulate the behavior of a spatially incoherent $\mathrm{x}$-ray source, was used to illuminate the sample. The pseudothermal $x$-ray source is produced by a monochromatic $\mathrm{x}$-ray beam passing through a slit array and a movable porous gold film deposited on a Si3N4 substrate. The monochromatic x-ray beam was
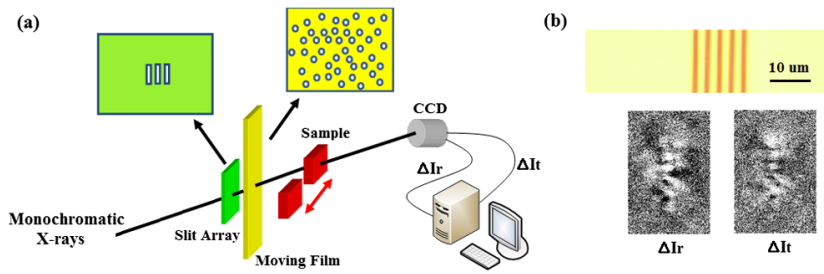

FIG. 2. Experimental setup for x-ray FGI using a pseudothermal $\mathrm{x}$-ray source is shown in (a). A monochromatic $\mathrm{x}$-ray beam passes through a slit array and a moving porous gold film to generate a controllable pseudothermal x-ray speckle pattern fluctuation, which is used in the experiment as the pseudothermal $\mathrm{x}$-ray source. The sample is moved in and out of the beam to get the intensity fluctuation $\Delta I_{t}\left(x_{t}\right)$ and $\Delta I_{r}\left(x_{r}\right)$, respectively, as required by the scheme of FGI without a beam splitter. (b) The optical microscope image of the sample and an example of intensity distribution pattern pairs $\Delta I_{t}\left(x_{t}\right)$ and $\Delta I_{r}\left(x_{r}\right)$ acquired in the $\mathrm{x}$-ray FGI experiment. 
produced by passing the $\mathrm{x}$ rays emitted from the synchrotron source through a double crystal monochromator with an energy resolution $\Delta E / E \approx 10^{-3}$. The flux of the $\mathrm{x}$ ray was $6.75 \times 10^{12}$ photons/sec and the energy was centered at $12.1 \mathrm{keV}(0.1 \mathrm{~nm}$ wavelength). The slit array is positioned in the optical path of the x-ray beam, and each slit of the array has a dimension less than or equal to the $\mathrm{x}$-ray coherent area at the slit array plane $(50 \mu \mathrm{m} \times 10 \mu \mathrm{m})$, so that each of the transmitted $\mathrm{x}$-ray subbeams passing through a slit is spatially coherent. The porous gold film with randomly distributed holes of diameter $<1 \mu \mathrm{m}$ was mounted on a computercontrollable translational device and placed closely behind the slit array; the depth of the holes were designed to be $\lambda / 2(n-1)=2.7 \mu \mathrm{m}$ (where $n$ is the refractive index of gold) to form a phase difference of $\pi$ between the area with and without holes for the $12.1 \mathrm{keV}$ hard $\mathrm{x}$ ray. After the bundle of spatially coherent $\mathrm{x}$-ray subbeams from the slit array passed through the porous gold film, chaotic distributed $\mathrm{X}$-ray speckle patterns are produced because of the spatially stochastic interference of the randomly modulated spatial coherent $\mathrm{X}$-ray subbeams from the porous gold film. The size of the porous gold film is much larger than the whole beam cross section of the monochromatic $\mathrm{x}$ rays; thus, when the porous gold film is moved transversely by the translational device to make the different part of the film illuminated, a pseudothermal $\mathrm{x}$-ray beam with chaotic fluctuating intensity distributions was produced and served as the incoherent x-ray source in our experiment. The CCD camera with effective pixel size of $0.37 \mu \mathrm{m} \times 0.37 \mu \mathrm{m}$ was placed $43 \mathrm{~cm}$ downstream from the gold film. The experimental sample was placed on a controllable translational stage, which can move the sample in and out of the pseudothermal x-ray beam as shown by the red arrow in Fig. 2(a), for performing the $\Delta I_{t}\left(x_{t}\right)$ and $\Delta I_{r}\left(x_{r}\right)$ measurements. In one measurement, a pair of x-ray signals, i.e., the intensity fluctuation of the testing beam $\Delta I_{t}\left(x_{t}\right)$ and that of the reference beam $\Delta I_{r}\left(x_{r}\right)$, was acquired as shown in Fig. 2(b). The porous gold film remained static in the acquisition period, and it was triggered to move transversely again after each of the measurements was completed. We should mention that the drift of the sample position relative to the illuminating pseudothermal $\mathrm{x}$-ray beam introduced by the shuttling was almost inevitable, but it barely influences the FGI system as demonstrated theoretically and experimentally in Ref. [39].

The sample in our experiment was a $2.2 \mu \mathrm{m}$ thick gold film with five slits on a Si3N4 substrate. The slits were separated by $d_{\text {slit }}=3 \mu \mathrm{m}$ and the width of each slit was $1 \mu \mathrm{m}$. Figure 2(b) shows the optical microscope image of the sample. Since the wavelength of the pseudothermal $\mathrm{x}$-ray source was $0.1 \mathrm{~nm}$, the intensity transmission and phase difference between the slits and the surrounding gold area was $53 \%$ and $0.81 \pi$, respectively. The distance from the sample to the CCD camera is $33 \mathrm{~cm}$ and the width of the sample illuminated by the $0.1 \mathrm{~nm}$ pseudothermal $\mathrm{x}$ rays is $13 \mu \mathrm{m}$, so the far-field diffraction condition
$\left(D^{2} / \lambda=13 \mu \mathrm{m}^{2} / 0.1 \mathrm{~nm}=1.69 \mathrm{~m}, D\right.$ is the total width of the five slits) is not satisfied. For FGI purpose, signals from only one fixed single pixel in the intensity pattern, recorded while the imaging example was placed in the beam, were needed to construct the correlation function. For convenience and better image quality (detailed in the Supplemental Material [40]) we recorded the full intensity pattern of the beam passing through the sample. An example of a pair of intensity distribution patterns is shown in Fig. 2(b). The exposure time in our experiment for each pattern was $3 \mathrm{sec}$, and the sample-translation time between two exposures was about $5 \mathrm{sec}$. The total acquisition time for each pair of the patterns was $16 \mathrm{sec}$. Obviously, the $\mathrm{x}$-ray intensity patterns display featureless random distributions, and no diffraction patterns of the sample can be directly observed.

The Fourier-transform diffraction pattern of the complex amplitude sample was obtained by calculating the correlation between the intensity fluctuations of the testing beam $\Delta I_{t}\left(x_{t}\right)$ and the reference beam $\Delta I_{r}\left(x_{r}\right)$ following Eq. (1). However, to improve the sampling efficiency in the calculation, we applied a reconstruction algorithm making use of sparsity constraints of the image [41] in reconstructing the Fouriertransform diffraction patterns. Figure 3(a) is the sample's diffraction pattern obtained by x-ray FGI with 284 pairs of measurement data used in the reconstruction calculation. The total acquisition time for the 284 pairs of intensity distribution patterns was about $76 \mathrm{~min}$ in the experiment. The cross-section curve of Fig. 3(a) is shown by the red line in Fig. 3(b), and the peak spacing of the red line in Fig. 3(b) is $11.1 \mu \mathrm{m}(0.37 \mu \mathrm{m} /$ pixel $\times 30$ pixels $=11.1 \mu \mathrm{m})$, which is in agreement with the theoretical value of the peak spacing $\left(\lambda d_{2} / d_{\text {slit }}=0.1 \mathrm{~nm} \times 33 \mathrm{~cm} / 3 \mu \mathrm{m}=11 \mu \mathrm{m}\right)$ predicted by Eq. (1). The blue line in Fig. 3(b) shows the numerical result of the sample's Fourier transformation, and it agrees well with the experimental result. Therefore, the sample's Fouriertransform diffraction pattern was obtained at the Fresnel region by $\mathrm{x}$-ray FGI, which is different from the case in $\mathrm{x}$-ray CDI, where the sample's Fourier-transform diffraction pattern should be obtained at far field.

By calculating the auto correlation of the intensity fluctuation of the testing beam $\Delta I_{t}\left(x_{t}\right)$, the Fourier-transform diffraction pattern of the squared modulus of the sample's transmittance can also be obtained [42]. The Fouriertransform diffraction pattern of the squared modulus of the sample's transmittance obtained in x-ray FGI and the corresponding cross-section curves are shown in Figs. 3(c) and 3(d), respectively. As Fig. 3(d) shows, the experimental result agrees well with the numerical Fourier transformation result. Thus, the amplitude and phase information of the complex amplitude sample were obtained separately from the autocorrelation of the intensity fluctuation of testing beam $\Delta I_{t}\left(x_{t}\right)$ and the correlation between the intensity fluctuations of the testing beam $\Delta I_{t}\left(x_{t}\right)$ and the reference beam $\Delta I_{r}\left(x_{r}\right)$ in our X-ray FGI experiment. 

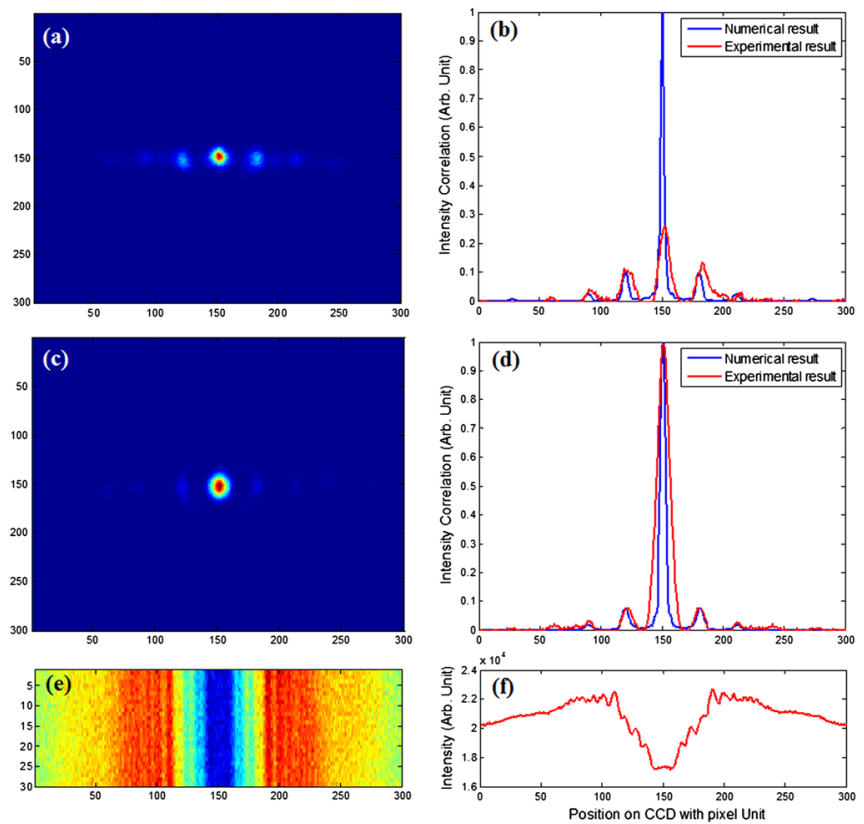

FIG. 3. Diffraction patterns of the sample obtained with x rays of $0.1 \mathrm{~nm}$ wavelength. (a) The Fourier-transform diffraction pattern of the sample's transmittance obtained by x-ray FGI, (c) the Fourier-transform diffraction pattern of the squared modulus of the sample's transmittance obtained in x-ray FGI, (e) the intensity distribution obtained by illuminating the sample directly with synchrotron $x$ rays. The red lines in (b),(d), and (f) are the cross-section curves of (a),(c) and (e), respectively. The blue lines in (b) and (d) are the corresponding numerical results obtained by the Fraunhofer diffraction integral.

For reference, the $\mathrm{x}$-ray intensity distribution recorded by the CCD camera when the sample was directly illuminated by the monochromatic $\mathrm{x}$-ray beam emitted from the synchrotron source through the double crystal monochromator is shown in false-color representation as Fig. 3(e). Figure 3(f) shows the cross-section curve of Fig. 3(e). By comparing Fig. 3(e) with Fig. 3(a) and Fig. 3(c), it can be found that the pattern obtained when illuminating the sample directly with monochromatic $\mathrm{x}$ rays is apparently different from the Fourier-transform patterns obtained in x-ray FGI.

Using a two-step phase-retrieval image reconstruction process based on FGI [43], in which first the amplitude part of the sample's transmittance is retrieved from the Fouriertransform patterns in Fig. 3(c), then combining the retrieved amplitude part of the sample's transmittance with the Fourier-transform pattern in Fig. 3(a), the phase part of the sample's transmittance was retrieved. The retrieved amplitude and phase distributions of the sample's transmittance are shown in Figs. 4(a) and 4(b), respectively. The maximum spatial frequency used in the reconstruction can be calculated as $q_{\max }=\left(0.37 \mu \mathrm{m} /\right.$ pixel $\times 300$ pixels $\left./ 2 \lambda d_{2}\right)$, so the pixel size in the retrieved image is $\left(1 / 2 q_{\max }\right)=$ $0.297 \mu \mathrm{m}$, and the separate distance between the slits in the

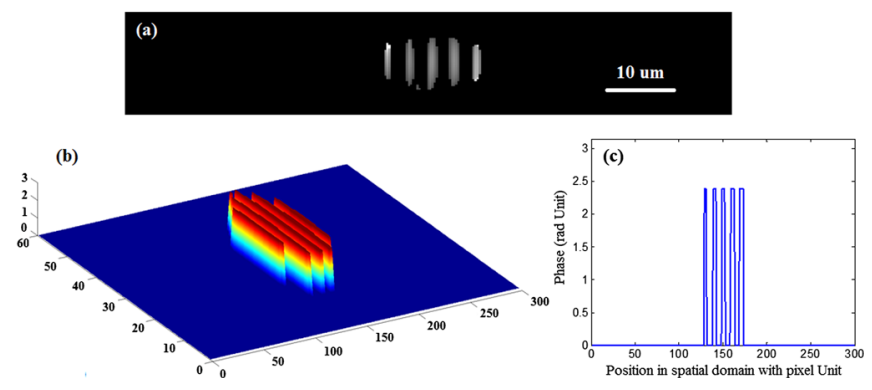

FIG. 4. The sample's distributions in the spatial domain retrieved from the Fourier-transform diffraction patterns obtained in X-ray FGI. (a) and (b) The amplitude and phase distributions of the sample's transmittance; (c) the profile of (b). The pixel size in the retrieved image is $0.297 \mu \mathrm{m}$.

retrieved image is $2.97 \mu \mathrm{m}(0.297 \mu \mathrm{m} /$ pixel $\times 10$ pixels $)$, which is in complete agreement with the spatial feature of the sample.

Our experimental results demonstrated for the first time that Fourier-transform ghost imaging can be achieved using pseudothermal hard $\mathrm{x}$ rays. The diffraction patterns are qualified enough to retrieve the amplitude and phase distributions of the sample in the spatial domain. The spatial resolution of FGI with incoherent $\mathrm{x}$ rays is determined by the maximum spatial frequency of the Fourier-transform diffraction pattern, which means the spatial resolution of lensless x-ray FGI is only limited by the wavelength theoretically, and provides the potential to achieve atomic resolution images of noncrystalline samples with laboratory $\mathrm{x}$-ray sources.

In summary, we have experimentally demonstrated Fourier-transform ghost imaging with pseudothermal hard $\mathrm{x}$ rays and a high resolution Fourier-transform diffraction pattern of the sample has been achieved at the Fresnel region by measuring the second-order intensity correlation of the lights, and the amplitude and phase distributions of the sample in the spatial domain have been retrieved successfully. This method extends $\mathrm{x}$-ray crystallography to noncrystalline samples and, as a lensless imaging scheme, the spatial resolution of $\mathrm{x}$-ray FGI is only limited by the wavelength in principle. An important feature of the x-ray FGI method is that it does not rely on a highly coherent $\mathrm{x}$-ray source to realize $x$-ray diffraction imaging, therefore, it provides a feasible way to achieve high resolution images of noncrystalline samples with widely accessible laboratory $\mathrm{x}$-ray sources. Eliminating the need for an intensive coherent source, FGI of not only bosons but also fermions such as neutrons and electrons, can also be expected; it provides a glimpse of the possibility of revolutionizing the current neutron and electron scattering methods widely used in research in materials science as well as biomedicine. Furthermore, the Fourier-transform diffraction pattern is acquired from correlated calculations, which removes the need for a beam stop and avoids missing low-frequency data in the diffraction patterns as in traditional x-ray CDI. Finally, 
the high frequency portion of the diffraction pattern that is out of the CCD detection area can also be captured by using the intensity distribution patterns that deviate from the center point in pairs [42], which means the spatial resolution of x-ray FGI systems can be doubly enhanced.

This work was supported by the National High Technology Research and Development Program of China under Grant Projects No. 2013AA122901 and No. 2013AA122902, the National Natural Science Foundation of China under Grant Project No. 11105205, and the Natural Science Foundation of Shanghai under Grant Project No. 09JC1415000.

Note added.-Recently, we became aware of related work [44].

*sshan@mail.shcnc.ac.cn

[1] Y. Shi, Cell 159, 995 (2014).

[2] B. Fleury, R. Cortes-Huerto, O. Taché, F. Testard, N. Menguy, and O. Spalla, Nano Lett. 15, 6088 (2015).

[3] D. V. Kosynkin, A. L. Higginbotham, A. Sinitskii, J. R. Lomeda, A. Dimiev, B. K. Price, and J. M. Tour, Nature (London) 458, 872 (2009).

[4] J. Miao, P. Charalambous, J. Kirz, and D. Sayre, Nature (London) 400, 342 (1999).

[5] H. N. Chapman et al., Nat. Phys. 2, 839 (2006).

[6] M. A. Pfeifer, G. J. Williams, I. A. Vartanyants, R. Harder, and I. K. Robinson, Nature (London) 442, 63 (2006).

[7] G. J. Williams, H. M. Quiney, B. B. Dhal, C. Q. Tran, K. A. Nugent, A. G. Peele, D. Paterson, and M. D. de Jonge, Phys. Rev. Lett. 97, 025506 (2006).

[8] B. Abbey, K. A. Nugent, G. J. Williams, J. N. Clark, A. G. Peele, M. A. Pfeifer, M. de Jonge, and I. McNulty, Nat. Phys. 4, 394 (2008)

[9] J. M. Rodenburg, A. C. Hurst, A. G. Cullis, B. R. Dobson, F. Pfeiffer, O. Bunk, C. David, K. Jefimovs, and I. Johnson, Phys. Rev. Lett. 98, 034801 (2007).

[10] P. Thibault, M. Dierolf, A. Menzel, O. Bunk, C. David, and F. Pfeiffer, Science 321, 379 (2008).

[11] H. N. Chapman et al., Nature (London) 470, 73 (2011).

[12] S. Boutet et al., Science 337, 362 (2012).

[13] A. V. Belinskii and D. N. Klyshko, J. Exp. Theor. Phys. 78, 259 (1994).

[14] T. B. Pittman, Y.H. Shih, D. V. Strekalov, and A. V. Sergienko, Phys. Rev. A 52, R3429 (1995).

[15] R. S. Bennink, S. J. Bentley, and R. W. Boyd, Phys. Rev. Lett. 89, 113601 (2002).

[16] R. S. Bennink, S. J. Bentley, R. W. Boyd, and J. C. Howell, Phys. Rev. Lett. 92, 033601 (2004).

[17] J. Cheng and S. Han, Phys. Rev. Lett. 92, 093903 (2004).
[18] A. Gatti, E. Brambilla, M. Bache, and L. A. Lugiato, Phys. Rev. Lett. 93, 093602 (2004).

[19] F. Ferri, D. Magatti, A. Gatti, M. Bache, E. Brambilla, and L. A. Lugiato, Phys. Rev. Lett. 94, 183602 (2005).

[20] G. Scarcelli, V. Berardi, and Y. Shih, Phys. Rev. Lett. 96, 063602 (2006).

[21] J. H. Shapiro, Phys. Rev. A 78, 061802 (2008).

[22] N. D. Hardy and J. H. Shapiro, Phys. Rev. A 87, 023820 (2013).

[23] M. Bina, D. Magatti, M. Molteni, A. Gatti, L. A. Lugiato, and F. Ferri, Phys. Rev. Lett. 110, 083901 (2013).

[24] E. Meyers, K. S. Deacon, and Y. Shih, Appl. Phys. Lett. 98, 111115 (2011).

[25] W. Gong, C. Zhao, H. Yu, M. Chen, W. Xu, and S. Han, Sci. Rep. 6, 26133 (2016).

[26] M. D’Angelo, M. V. Chekhova, and Y. Shih, Phys. Rev. Lett. 87, 013602 (2001).

[27] J. Xiong, D. Z. Cao, F. Huang, H. G. Li, X. J. Sun, and K. Wang, Phys. Rev. Lett. 94, 173601 (2005).

[28] Y. H. Zhai, X. H. Chen, D. Zhang, and L. A. Wu, Phys. Rev. A 72, 043805 (2005).

[29] R. F. Liu, P. Zhang, Y. Zhou, H. Gao, and F. L. Li, Sci. Rep. 4, 4068 (2014).

[30] W. Gong and S. Han, Sci. Rep. 5, 9280 (2015).

[31] W. Gong and S. Han, Phys. Lett. A 376, 1519 (2012).

[32] H. Wang, S. Han, and M. I. Kolobov, Opt. Express 20, 23235 (2012)

[33] B. Sun, M. P. Edgar, R. Bowman, L. E. Vittert, S. Welsh, A. Bowman, and M. J. Padgett, Science 340, 844 (2013).

[34] M. Zhang, Q. Wei, X. Shen, Y. Liu, H. Liu, J. Cheng, and S. Han, Phys. Rev. A 75, 021803 (2007).

[35] H. Liu, X. Shen, D. M. Zhu, and S. Han, Phys. Rev. A 76, 053808 (2007).

[36] H. Liu, J. Cheng, and S. Han, Opt. Commun. 273, 50 (2007).

[37] R. Cerbino, L. Peverini, M. A. C. Potenza, A. Robert, P. Bösecke, and M. Giglio, Nat. Phys. 4, 238 (2008).

[38] D. Magatti, A. Gatti, and F. Ferri, Phys. Rev. A 79, 053831 (2009).

[39] C. Zhang, W. Gong, and S. Han, Appl. Phys. Lett. 102, 021111 (2013).

[40] See Supplemental Material at http://link.aps.org/ supplemental/10.1103/PhysRevLett.117.113901 for the method which improves the image quality by using multi-point intensity data at the detector of the testing beam in X-ray Fourier-transform ghost imaging.

[41] H. Wang and S. Han, Europhys. Lett. 98, 24003 (2012).

[42] M. Zhang, Q. Wei, X. Shen, Y. Liu, H. Liu, Y. Bai, and S. Han, Phys. Lett. A 366, 569 (2007).

[43] G. Ying, Q. Wei, X. Shen, and S. Han, Opt. Commun. 281, 5130 (2008).

[44] D. Pelliccia, A. Rack, M. Scheel, V. Cantelli, and D. M. Paganini, following Letter, Phys. Rev. Lett. 117, 113902 (2016). 